\title{
Management of foreign body aspiration or ingestion in dentistry
}

\author{
Parolia $\mathbf{A}^{1}$, Kamath $\mathbf{M}^{2}$, Kundubala $\mathbf{M}^{3}$, Manuel TS ${ }^{1}$, Mohan $\mathbf{M}^{4}$
}

${ }^{1}$ Assistant Professor, ${ }^{3}$ Professor and HOD, Department of conservative Dentistry and Endodontics, ${ }^{4}$ Assistant professor, Department of Prosthodontics, MODS, Mangalore, India, ${ }^{2}$ Assisstant Professor, Department of Cardiothoracic Surgery KMC, Manipal, India.

\begin{abstract}
Foreign body aspiration or ingestion can be a potential complication during routine dental treatment. The handling of dental objects requires particular care, especially where the patient is supine or semi-recumbent. Dentist must be able to manage emergency situations in which patients accidentally swallow dental instruments or materials during treatment and procedures. This article reviews the complications, management and prevention of aspiration or ingestion of foreign bodies.
\end{abstract}

Key words: Foreign bodies, Aspiration/ Ingestion, Management, Prevention

$\mathrm{T}^{\mathrm{s}}$ he ingestion of foreign bodies is most commonly a problem in young children ${ }^{1}$. In adults, it occurs accidentally more commonly among those with psychiatric disorder, mental retardation, use of local anaesthetics, and altered consciousness associated with intravenous sedaion ${ }^{2}$. Swallowing of dental materials and devices may lead to serious complication during dental treatment. Any object routinely placed into or removed from the oral cavity during dental or surgical procedures can be aspirated or swallowed. These items can include teeth, restorations, restorative materials, instruments, implant parts, rubber dam clamps, gauze packs and impression materials ${ }^{3,4,5,6,7}$. The majority of foreign bodies that reach the gastrointestinal tract pass spontaneously. However, $10-20 \%$ of cases require nonsurgical intervention, while $1 \%$ or less may require surgery. Patients swallowing foreign bodies are usually asymptomatic but symptoms may arise later. Foreign body aspiration or ingestion may cause damage to gastric mucosa, septic abscess, intestinal perforations, partial or complete airway obstruction, post obstructive pneumonia, respiratory distress, pnuemothorax or haemorrhage ${ }^{8,9,10}$. If these cases are not properly managed and timely intervention is not carried out, it can be lethal ${ }^{11}$. Therefore, general dental practitioners should be aware of a protocol of management and prevention of swallowing or aspiration of dental objects.

\section{Signs and symptoms}

This is highly variable and depends on whether it is a child or an adult. In children the event may have been witnessed, reported by the child, or be suspected/ discovered subsequently when a child becomes ill. Any symptoms or signs are also largely dependent on where the object is impacted. About $75 \%$ of children who have an impacted foreign body will have it at the level of the upper oesophageal sphincter while roughly $70 \%$ of affected adults having impaction at the level of the lower oesophageal sphincter.

\section{Foreign bodies at Oropharyngeal level}

- Overall, about $60 \%$ of foreign bodies become trapped at this level (commonly at, or just below, the level of the cricopharyngeus muscle).

- Patients usually have a clear sensation of something being trapped that is relatively well localised.

- There is usually discomfort ranging from mild to quite severe.

- Drooling of saliva and an inability to swallow may be present.

- Airway compromise may occur if large objects are trapped.

- A delayed presentation with infection or perforation may occur with objects that

- Stuck at this level.

\section{Foreign bodies at Oesophageal level}

- In adults there is usually an acute presentation following ingestion of an object or food item that gets stuck.

\section{Correspondence}

Dr. Abhishek Parolia

Assistant Professor, Department of conservative Dentistry and Endodontics,

MCODS, Mangalore,India

E-mail: paroliaabhi@yahoo.com 
- There tends to be a vague sensation of something being stuck in the centre of the chest or epigastric region, indicating that the object is probably at the level of the aortic cross-over or the lower oesophageal sphincter.

- There may be dysphagia for the remainder of the meal prompting presentation or salivary pooling/drooling if there is complete oesophageal obstruction.

- Gagging, vomiting, retching, neck and/or throat pain are more common presentations in children with oesophageal foreign bodies.

- Children with partial oesophageal obstruction may present with a chronic course featuring inability to feed, failure to thrive, fever, recurrent aspiration pneumonitis/pneumonia or respiratory embarrassment/stridor (due to tracheal impingement).

\section{Foreign bodies at Sub-oesophageal level}

It may present with a range of symptoms depending on the degree of progression of the object through the gut. Vague symptoms such as abdominal distension and discomfort, fever, recurrent vomiting, passing rectal blood/melaena and/or other symptoms of acute or subacute intestinal obstruction may be present.

\section{Symptoms due to gastrointestinal perforation}

If an object perforates the oesophagus it tends to cause acute mediastinitis with chest pain, dyspnoea and severe odynophagia (pain associated with swallowing), along with signs of pneumonitis/pleural effusion ${ }^{5}$. Perforation below the level of the oesophagus will cause symptoms and signs of acute/sub-acute peritonitis.

\section{Examination of the patient with definite/suspected} foreign body ingestion/entrapment

Careful examination should be carried out for clinical and medicolegal reasons.

- Assess the airway and respiratory function to exclude/highlight any compromise

- Check vital signs to exclude impending catastrophic presentation due to airway obstruction or acute gastrointestinal perforation, or fever in case of delayed presentation

- Open the mouth and observe the oropharynx with a bright light

- Consider indirect laryngoscopy and/or fibreoptic examination of the pharynx if dentist has appropriate equipment and a sufficiently experienced practitioner available

- Gently palpate the neck and assess tracheal position/ compression
- Formally examine the chest and listen to the lungs

- Perform a cardiovascular examination

\section{Investigation methods}

\section{Abdominal and Chest $X$-rays:}

When there is a history of a swallowed or aspirated radio-opaque object, then abdominal x-ray (Fig.1), posteroanterior chest X-ray (Fig.2, 3) and lateral chest X-ray (Fig.4, 5) should be carried out to confirm or refute the possibility of oesophageal entrapment.

\section{Endoscopy}

Endoscopy of upper gastrointestinal tract should be carried out when the ingested object is not radio-opaque, $\mathrm{x}$-ray investigations are unlikely to help.

Urgent endoscopy is mandatory in cases where there is airway obstruction or evidence of other severe complications.

Endoscopy is definitely indicated when ingested objects are sharp, non-radio-opaque, elongated, or where there are multiple swallowed objects or a high-risk of oesophageal injury.

Endoscopy is also indicated for gastric or proximalduodenal foreign bodies that have a diameter of $2 \mathrm{~cm}$, length of $5-7 \mathrm{~cm}$ or are eccentrically-shaped and prone to enlodgement and perforation, such as open safety pins.

Endoscopy is a relatively safe procedure in experienced hands, but costly, and should therefore be avoided as a routine intervention if possible.

\section{CT scans}

CT scanning of thorax/abdomen is highly useful at locating entrapped objects of various types and considered superior by many to plain x-ray imaging.

CT scanning is the investigation of choice if there is reason to suspect perforation or abscess formation.

\section{Complications due to ingestion/ aspiration}

\section{- Oropharyngeal foreign bodies}

O Scratches and lacerations of oropharyngeal mucosa

○ Perforation

O Retro-pharyngeal abscess

O Soft-tissue infection or abscess

- Oesophageal foreign bodies

○ Scratches, lacerations or abrasions of mucosa 

O Oesophageal necrosis
○ Retropharyngeal abscess
O Oesophageal stricture
O Oesophageal perforation and subsequent paraoesophageal abscess
○ Mediastinitis
O Pneumothorax and/or pneumomediastinum
O Pericarditis/cardiac tamponade
○ Tracheoesophageal fistula

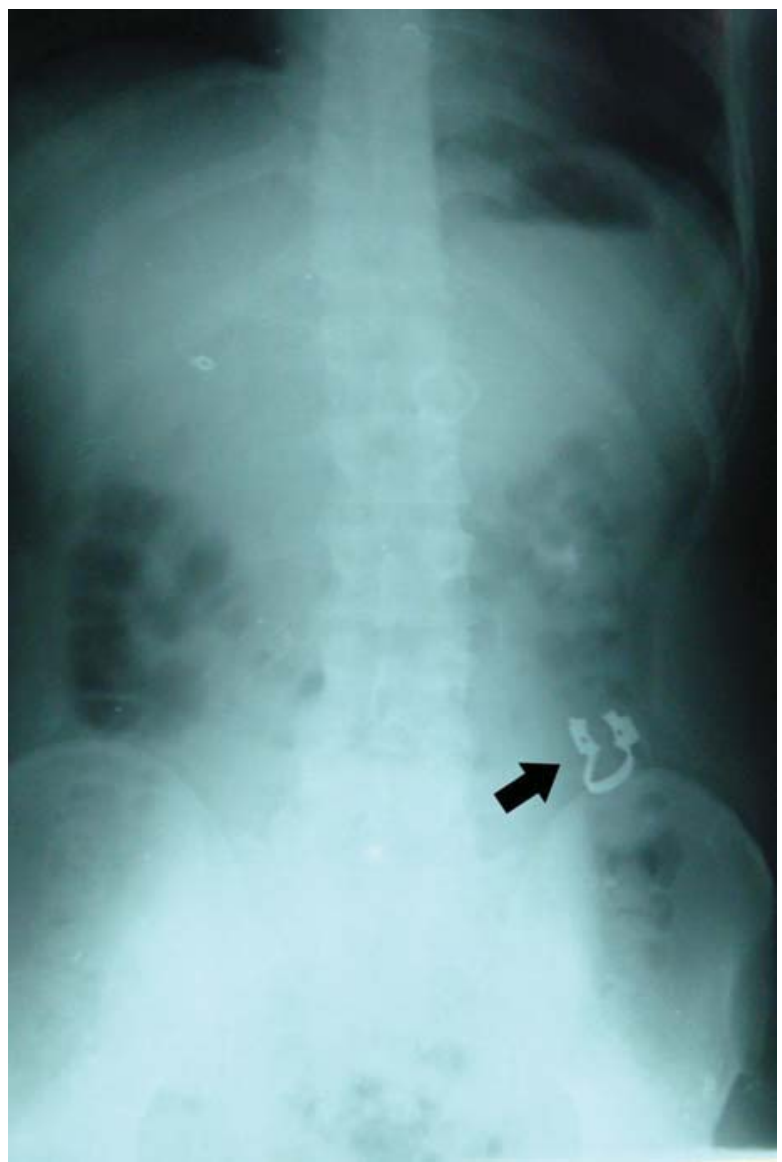

Fig 1: Abdominal X-ray showing rubber dam clamp in large intestine
O Aorto-oesophageal fistulae or other mediastinal vascular injury

\section{- Gastric/small-intestine foreign bodies:}

○ Entrapment of object within Meckel's diverticulum

O Perforation leading to peritonitis and advanced sepsis

○ Acute or sub-acute small-intestinal obstruction

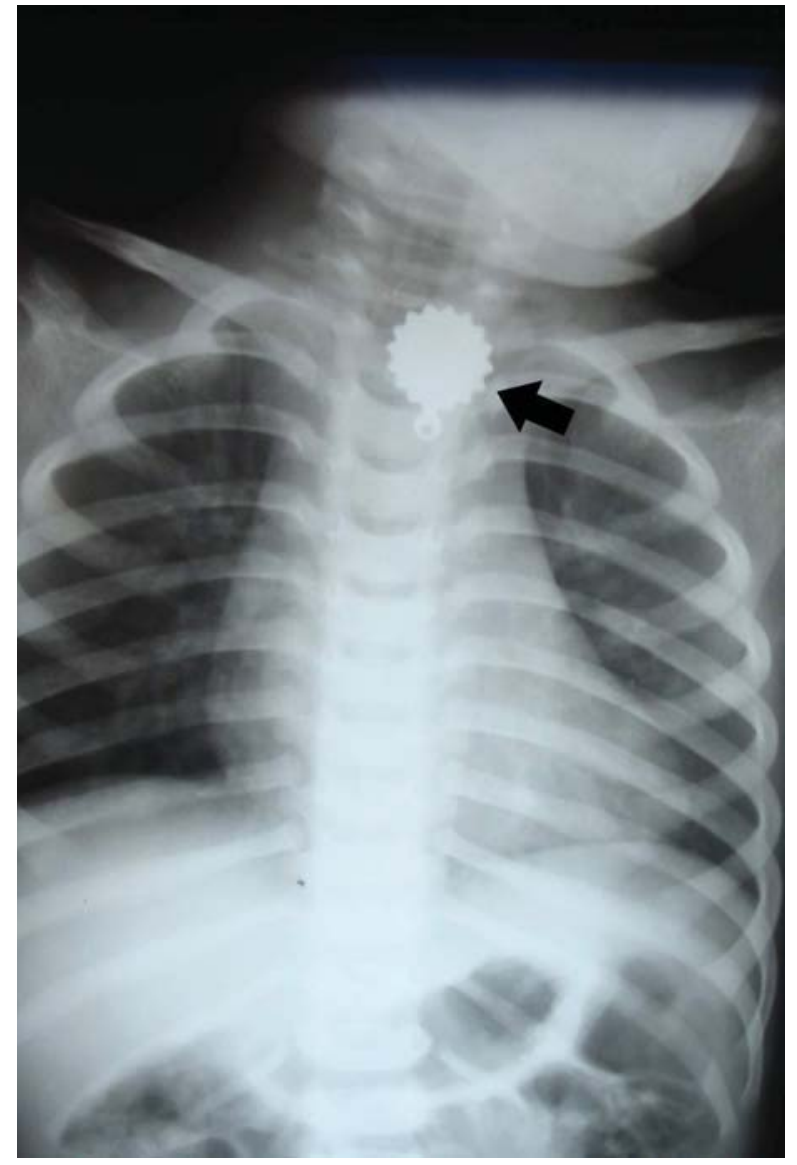

Fig 2: Postero-anterior chest $\mathrm{x}$-ray showing pendant in oesophagus 


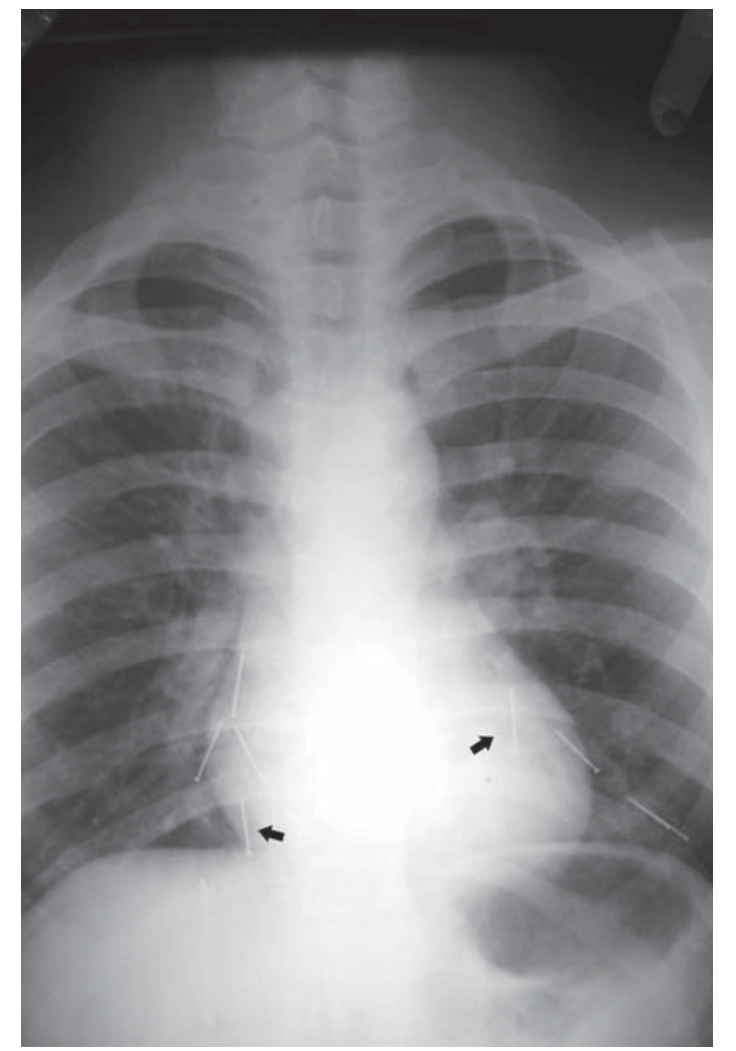

Fig 3: Postero-anterior chest X-ray showing multiple pins

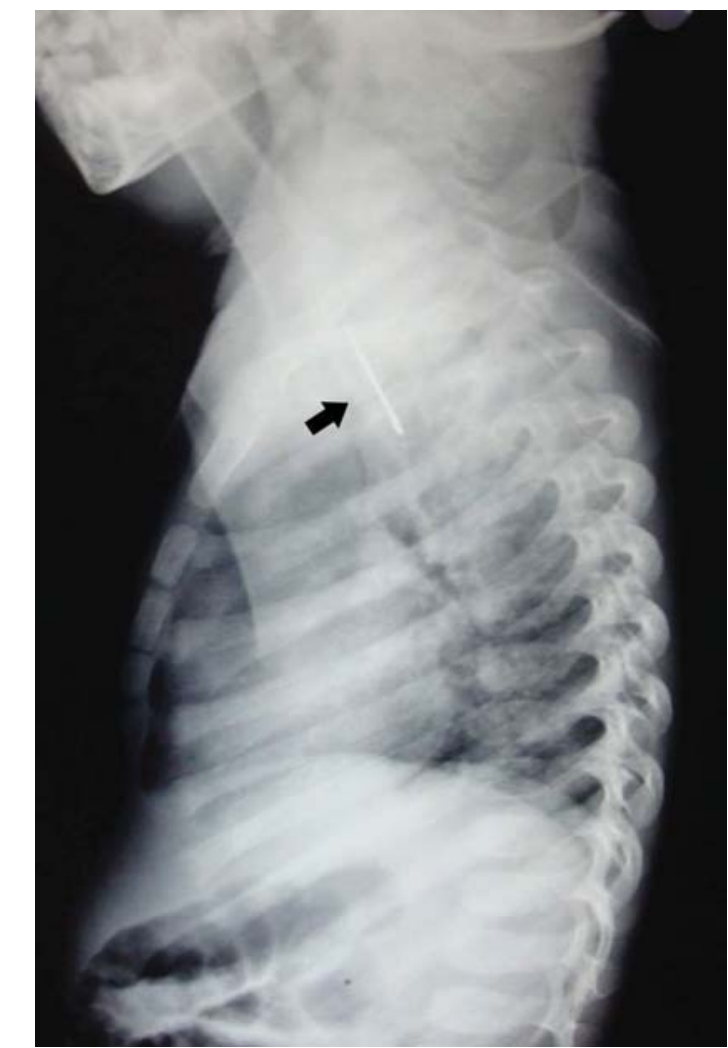

Fig 4 :Lateral chest $x$-ray showing pendant in oesophagus

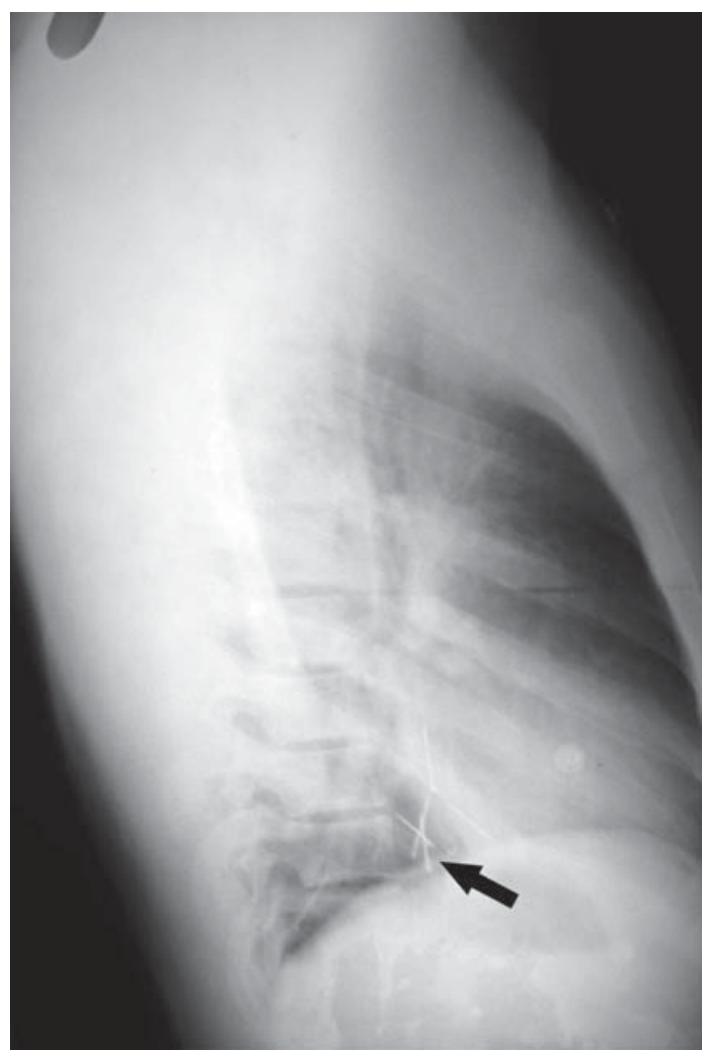

Fig 5 : Lateral chest x-ray showing multiple pins 
Flow chart for management of swallowed/ aspirated dental objects

Incident occurs

\section{Maintain patient in reclined position}

$\downarrow$

Airway not compromised

Examination of mouth and local area

$\downarrow$

Potentially swallowed / Not swallowed

$\begin{array}{cc}\text { Maintain airway } & \text { Retrieve and identify the object } \\ \downarrow & \downarrow\end{array}$

Reassure the patient
Airway compromised

$\downarrow$

Ask patient to cough

$\downarrow$

Object not retrieved and airway obstruction exists

$\downarrow$

Perform Heimlich manoeuvre

$\downarrow$

Immediate summon, Support including cricothyroidotomy where necessary

\section{$\downarrow$ \\ Identify the location of object

$\begin{array}{cc}\downarrow & \downarrow \\ \text { Gastroinestinal tract } & \text { Oesophagus } \\ \downarrow & \downarrow \\ \text { Refer to gastroenterologist } & \text { Endoscopic removal }\end{array}$

Escort patient to hospital for clinical/ redigraphical examination

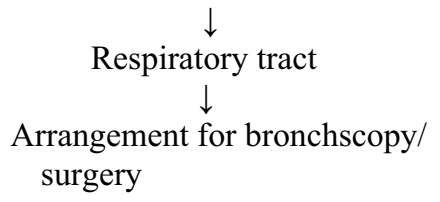

Monitor 2 weeks, examination of stools

$\downarrow$

Object not retrieved

$\downarrow$

Radiographic examination

$\downarrow$

Object still present $\quad$ Object no longer present (assume object passed, reassure patient)

Consider need for endoscopy or surgery

\section{Management}

Many reports in literature describe accidental ingestion or aspiration of dental instruments, restorations and prosthesis during dental treatment ${ }^{12,13,14,15,16}$. Any patient may swallow or aspirate foreign objects, but the risk is greater in elderly patients and those under the effect of narcotics, sedation or nitrous oxide because of diminished protective reflexes ${ }^{17}$. There is no consensus in the literature regarding the position of the patient to minimize the risk of foreign bodies aspiration. Neuhauser ${ }^{18}$ suggested that patients in a supine position are more or less prevented from swallowing foreign objects. Barkmeier et $\mathrm{al}^{19}$ stated that supine position increases the risk of swallowing.

When any dental instrument is aspirated / swallowed :

- Act quickly to locate and remove any object that may be causing acute upper airway obstruction. Keep the patient's head low, turn it to the side, and ask the patient to cough. Administer sharp blows on the patient's back.

- If object is visible, grasp it with small forceps, or use a suction tip, being careful not to push it deeper into the throat. 
- If it is not possible to grasp the object, or if it is evident that the object is lodged in the airway (difficulty in breathing), the Heimlich manoeuvre ${ }^{20}$ may be attempted. In the Heimlich manoeuvre, place both hands one on top of the other, over the abdominal cavity just below the ribs and press forcefully, using the remaining air in the patient's lung to pop out the obstruction, this manoeuvre should not be used if there is a possibility of the foreign body being swallowed. To do so may result injury to the oesophagus or stomach.

- Aspiration of objects into the airway is usually presents a more dangerous situation than does swallowing. Foreign body aspiration usually involves the right bronchus in adults because of the anatomic configuration ${ }^{8}$.

- Where airway obstruction is life-threatening and an object cannot be removed then obtain urgent senior anaesthetic/ENT advice and/or consider cricothyroidotomy, as a life-saving procedure.

- Patients outside of hospital with significant airway/GI obstruction should be transferred as an emergency, in a sitting position, with a suction catheter available for them to use to remove obstructed saliva.

- Children with upper gastrointestinal obstruction and/or airway compromise should be allowed to stay in their parent's arms whilst being transferred to, or assessed in, hospital, to reduce anxiety and worsening airway embarrassment.

\section{Indications of instability or a need for urgent transfer to hospital include:}

- Airway compromise

- Drooling

- Inability to swallow fluids

- Sepsis

- Suspicion of intestinal perforation

- Evidence of active bleeding

- Those with objects lodged in the oesophagus will usually require some form of intervention to prevent ulceration and/or other complications; options include endoscopy, removal with a catheter and medical therapy to dilate the lower oesophageal sphincter.

- Stable patients who have swallowed small, smooth objects, who have no evidence of oesophageal entrapment, otherwise negative imaging, with no evidence of damage can often be managed conservatively with follow-up at 24 hours or so to check that they remain well; passage of objects in stool may take days to weeks and parents should observe for their presence.
- Patients with stomach or small-intestine foreign bodies of width $<2 \mathrm{~cm}$ or length $<6 \mathrm{~cm}$ can be discharged home with instructions on symptoms that should prompt their re-attendance; patients with larger or sharp objects in these areas should be referred to a gastroenterologist who may carry out serial x-rays.

- A diet high in roughage and frequent ingestion of soft food items, like banana or moistened bread, may aid in the passage of swallowed foreign object.

\section{Prevention}

There are several strategies to avoid aspiration of objects during routine dental treatment. The easiest and most common procedure for routine restorative and endodontic procedures is the use of rubber dam ${ }^{21,22}$. It offers effective protection against aspiration or swallowing of endodontic instruments, broken burs, restorative materials and pins. While the rubber dam reduces the risk of aspiration during restorative procedures, it is possible for the dam clamp itself to be aspirated. To reduce this risk, dental floss should be tied to secure rubber dam clamp ${ }^{14}$. Many dental techniques preclude the use of the rubber dam, particularly during routine oral surgery and prosthodontic procedures. An alternative is to place a $4 \times 4$ inch gauze protective barrier in the oral cavity distal to the area where small items are being manipulated. The dentist may also prevent cast restoration being aspirated by using dental floss $^{23,24}$. Dentist should also instruct patients that if an object falls on the tongue, they should try to suppress the swallowing reflex and turn their heads to the side. One prosthetic procedure that does not easily allow for the above barrier techniques is full arch impressions. An impression procedure may put a patient at a risk of aspirating the impression material if a large amount of material and/ or low viscosity material is introduced to the posterior oral cavity. Therefore, use of the most viscous material available that will achieve the desired level of accuracy for the impression procedure is recommended ${ }^{9}$.

\section{Strategies to prevent aspiration}

- Use a rubber dam

- Use a gauze throat pack

- Use high velocity evacuation

- Use Washfield technique

- Use a high viscosity type of impression material

- Use a custom tray, with an open palate design for maxillary arch impression

- Observe the entire impression procedure

- Use a more upright position if possible

- Provide thorough instructions to the patients 


\section{Conclusion}

In summary general dental practitioners must be aware of a correct protocol to manage those patients who are suspected of having ingested/ aspirated a foreign body. They should also take necessary steps to avoid such emergencies during routine dental treatment.

\section{References}

1. Webb WA. Management of foreign bodies of the upper gastrointestinal tract. Gastroenterology. 1988; 94:204-16.

2. Gitlin DF, Caplan JP, Rogers MP, et al; Foreignbody ingestion in patients with personality disorders. Psychosomatics. 2007; 48:162-6.

3. Leith R, Fleming P, Redahan S, Doherty P. Aspiration of an avulsed primary incisor: a case report. Dent Traumatol. 2008; 24:24-6.

4. Holan G, Ram D. Aspiration of an avulsed primary incisor. A case report. Int $\mathrm{J}$ Paediatr Dent. 2000; 10:150-2.

5. Tiwana KK, Morton T, Tiwana PS. Aspiration and ingestion in dental practice: a 10-year institutional review. J Am Dent Assoc. 2004; 135:1287-91.

6. HillEE, RubelB.Apractical review of prevention and management of ingested/aspirated dental items. Gen Dent. 2008; 56:691-4.

7. Fredekind RE, McConnell TA, Jacobsen PL. Ingested objects: a case report with review of management and prevention. J Calif Dent Assoc. 1995; 23:50-5.

8. Basoglu Ok, Budendi N, Caririci U, Turhar K Aysan T. Pulmonary aspiration of a two unit bridge during a deep sleep. J Oral Rehab. 2005; 32:461-3.

9. Kimberley DR. Unrecognized aspiration of mandibular incisor. J Oral Maxillofac Surg. 2001; 59: 350-2.

10. Fields RT, Schow SR. Aspiration and ingestion of foreign bodies in oral and maxillofacial surgery; a review of literature and report of five cases. J Oral Maxillofac Surg. 1998; 56: 1091-8.

11. Karkar RA, Ayyar VB. Aetiological aspects of lung abscess. J Post Med. 1981; 27: 163-6.
12. Rizzalti-Barbosa CM, Cunha FL, Bianchini WA, Albergaria-Barbosa JR, Gomes BP. Accidental impaction of a unilateral removable partial denture: a clinical report. J Prosthet Dent. 1999; 82:270-1

13. Weimann MM, Weimann DS, Lingle DM, Brosnan KM, Santora TA, Report of an aspirated gold crown utilizing the laproscopic biopsy forceps: a report. Quintessence Int. 1995; 26: 211-3.

14. Cameron SM, Whitlock WL, Tabor MS. Foreign body aspiration in dentistry: a review. J Am Dent Assoc. 1996; 127: 1224-9.

15. Milton TM, Hearing SD, Ireland AJ. Ingested foreign bodies associated with orthodontic treatment: report of three cases and review of ingestion/ aspiration incident management. $\mathrm{Br}$ Dent J. 2001; 190:592-6.

16. Auluck A, Desai R. Accidental swallowing of a prosthesis. Dent Update. 2008; 35: 577-9.

17. Mubin U, Tokassavul S. Preventing aspiration or ingestion of fixed restorations. J Prosthet Dent. 2003; 89:223-4.

18. Neuhauser W. Swallowing of a temporary bridge by a reclining patient being treated by a seated dentist. Quintessence Int Dent Dig. 1997; 6: 9-10.

19. Barkmeier WW, Cooley RL, Abrams H. Prevention of swallowing or aspiration of foreign objects. J Am Dent Assoc. 1978; 97: 473.

20. Heimlich HJ. The Heimlich manoeuvre, prevention of death from choking on foreign bodies. J Occup Med. 1977; 19: 208-10.

21. Stewardson DA, McHugh ES. Patient's attitudes to rubber dam. Int Endod J. 2002 ;35: 812-9.

22. Lynch CD, McConnell RJ. Attitudes and use of rubber dam by Irish general dental practitioners. Int Endod J. 2007; 40: 427-32.

23. Al-Rashed MA. A method to prevent aspiration or ingestion of cast post and core restorations. J Prosthet Dent. 2004; 91: 501-2.

24. Jacob R, Shillenberg HT. A method to prevent swallowing or aspiration of cast restorations. J Prosthet Dent. 1981; 46: 642-5. 